\title{
Global analysis of nucleon strange form factors at low $Q^{2}$
}

\author{
Jianglai Liu, ${ }^{*}$ Robert D. McKeown, and Michael J. Ramsey-Musolf ${ }^{\dagger}$ \\ W. K. Kellogg Radiation Laboratory, California Institute of Technology, Pasadena, California 91125, USA
}

(Received 1 June 2007; published 2 August 2007)

\begin{abstract}
We perform a global analysis of all recent experimental data from elastic parity-violating electron scattering at low $Q^{2}$. The values of the electric and magnetic strange form factors of the nucleon are determined at $Q^{2}=0.1 \mathrm{GeV} / c^{2}$ to be $G_{E}^{s}=-0.008 \pm 0.016$ and $G_{M}^{s}=0.29 \pm 0.21$.

DOI: 10.1103/PhysRevC.76.025202

PACS number(s): 11.30.Er, 13.40.Gp, 13.60.Fz
\end{abstract}

The existence of a "sea" of quarks and antiquarks in the nucleon has been firmly established in deep-inelastic lepton scattering experiments as well as in the production of dilepton pairs (the Drell-Yan process). However, demonstrating the role of these $\bar{q} q$ pairs in the static electromagnetic properties of the nucleon has been a more elusive and difficult task.

As the lightest quark that contributes only to the $q \bar{q}$ sea, the strange quark provides a unique window on the role of the sea in the nucleon's electromagnetic structure. As suggested by Kaplan and Manohar [1], knowledge of neutral current form factors, when combined with electromagnetic form factors, provides access to the contribution of strange quarks to these form factors. At low momentum transfers, the neutral current form factors can be determined through parity-violating (PV) electron scattering experiments [2,3].

During the last decade, there has been dramatic progress in the study of the strange quark-antiquark contributions to the nucleon elastic electromagnetic form factors. A series of definitive PV electron scattering experiments along with several theoretical studies now provides a basis for extracting precision information on these strange quark contributions. In this work we report the results of a global analysis of all these experiments, including both the latest data obtained in experiments performed at the Jefferson Laboratory and the appropriate theoretical input on radiative corrections, and obtain values for the strange electric and magnetic form factors of the nucleon at a four-momentum transfer $Q^{2}=0.1 \mathrm{GeV} / c^{2}$. We have also studied the sub-leading $Q^{2}$ dependence of these two form factors and found that so far the data do not provide conclusive information.

\section{STRANGE FORM FACTORS AND PARITY-VIOLATING ELECTRON SCATTERING}

The nucleon vector strange form factors, $G_{E}^{s}$ and $G_{M}^{s}$, characterize the contribution of the strange sea quarks to the nucleon electromagnetic form factors, and thereby their contribution to the charge and magnetization distributions in the nucleon. With polarized electron facilities, $G_{E}^{s}$ and $G_{M}^{s}$ can be accessed by measuring the PV asymmetries in

\footnotetext{
*jliu@caltech.edu

†On leave from the Department of Physics, University of Wisconsin-Madison, Madison, WI 53706, USA.
}

elastic $e-p$ scattering, quasielastic $e-d$ scattering, and elastic $e-{ }^{4} \mathrm{He}$ scattering [4]. In very general terms, the parity-violating asymmetry $A_{\mathrm{PV}}$ can be written as

$$
A_{\mathrm{PV}}=A_{\mathrm{nvs}}+\eta_{E} G_{E}^{s}+\eta_{M} G_{M}^{s},
$$

where $A_{\text {nvs }}$ is the "non-vector-strange" asymmetry (independent of $G_{E}^{s}$ and $G_{M}^{s}$ ) and $\eta_{E}$ and $\eta_{M}$ are functions of kinematic quantities, nucleon electromagnetic form factors, and nuclear models (for nonhydrogen targets).

For elastic $e$ - $p$ scattering, the full form of the asymmetry is [4]

$$
\begin{aligned}
A_{\mathrm{PV}}^{p}= & -\frac{G_{F} Q^{2}}{4 \sqrt{2} \pi \alpha} \frac{1}{\left[\epsilon\left(G_{E}^{p}\right)^{2}+\tau\left(G_{M}^{p}\right)^{2}\right]} \\
& \times\left\{\left(\epsilon\left(G_{E}^{p}\right)^{2}+\tau\left(G_{M}^{p}\right)^{2}\right)\left(1-4 \sin ^{2} \theta_{W}\right)\left(1+R_{V}^{p}\right)\right. \\
& -\left(\epsilon G_{E}^{p} G_{E}^{n}+\tau G_{M}^{p} G_{M}^{n}\right)\left(1+R_{V}^{n}\right) \\
& -\left(\epsilon G_{E}^{p} G_{E}^{s}+\tau G_{M}^{p} G_{M}^{s}\right)\left(1+R_{V}^{(0)}\right) \\
& \left.-\epsilon^{\prime}\left(1-4 \sin ^{2} \theta_{W}\right) G_{M}^{p} G_{A}^{e}\right\}
\end{aligned}
$$

with

$$
\begin{aligned}
\tau & =\frac{Q^{2}}{4 M_{p}^{2}}, \quad \epsilon=\left(1+2(1+\tau) \tan ^{2} \frac{\theta}{2}\right)^{-1}, \\
\epsilon^{\prime} & =\sqrt{\tau(1+\tau)\left(1-\epsilon^{2}\right)},
\end{aligned}
$$

where $M_{p}$ is the mass of the proton and $\theta$ is the electron scattering angle. In Eq. (2), $G_{F}$ and $\alpha$ are the Fermi and fine structure constants, respectively. $Q^{2}$ is the four-momentum transfer. $G_{E, M}^{(p, n)}$ are the proton and neutron electric and magnetic form factors, while $G_{A}^{e}$ is the proton axial form factor seen by an electron. To extract contributions from $G_{E, M}^{s}$ to $A_{\mathrm{PV}}^{p}$, one must include the effects of Standard Model (SM) $\mathcal{O}(\alpha)$ electroweak radiative corrections [5]. It is often useful to characterize these corrections in terms of ratios $R_{V, A}$ of the $\mathcal{O}(\alpha)$ hadronic vector $(V)$ and axial vector $(A)$ weak neutral current amplitudes to the corresponding tree-level amplitudes. The $R_{V}^{p}, R_{V}^{n}$, and $R_{V}^{(0)}$ give these ratios for vector proton, neutron, and SU(3)-singlet amplitudes, respectively. In principle, their values can be obtained using the SM predictions for the effective electron-quark couplings $C_{1 q}$ given in Ref. [8]. However, the quoted $C_{1 q}$ do not include perturbative QCD contributions or coherent strong interaction effects in the radiative corrections associated with elastic scattering from a nucleon. A recent analysis of these effects has been given in 
TABLE I. Summary of parameters (with some uncertainties in parentheses) in Eqs. (2), (3), and (4). The values of $\alpha, \hat{s}_{Z}^{2}, G_{F}, M_{p}, g_{A} / g_{V}$ are taken directly from Ref. [8]. $R_{V}^{n}$, and $R_{V}^{(0)}$ are converted from $C_{1 q}$ parameters in Ref. [8]. $R_{V}^{p}$ is derived from the proton weak charge given in Ref. [10]. We adopt the value and uncertainty of $\Lambda_{A}^{2}$ from Ref. [11], $3 F-D$ from Ref. [12], and $\Delta s$ from Ref. [13].

\begin{tabular}{lc}
\hline \hline Parameter & Value \\
\hline$\alpha$ & $1 . / 137.03599911$ \\
$\hat{s}_{Z}^{2}$ & $0.23122(15)$ \\
$G_{F}$ & $1.16637 \times 10^{-5} / \mathrm{GeV}^{2}$ \\
$M_{p}$ & $0.98272 \mathrm{GeV}$ \\
$\Lambda_{A}^{2}$ & $1.00(0.04)(\mathrm{GeV} / \mathrm{c})^{2}$ \\
$g_{A} / g_{V}$ & -1.2695 \\
$3 F-D$ & $0.58(0.12)$ \\
$\Delta s$ & $-0.07(0.06)$ \\
$R_{V}^{p}$ & -0.0520 \\
$R_{V}^{n}$ & -0.0123 \\
$R_{V}^{(0)}$ & -0.0123 \\
\hline \hline
\end{tabular}

Ref. [9] and updated in Ref. [10]. The latter work also gives an improved treatment of strong interaction contributions to the running of the weak mixing angle in the $\overline{M S}$ renormalization scheme from its value at the $Z$ pole, $\hat{s}_{Z}^{2} \equiv \sin ^{2} \hat{\theta}_{W}\left(M_{Z}\right)$, to the quantity appropriate for precise, low-energy neutral current experiments, $\sin ^{2} \hat{\theta}_{W}(0)$. All of these effects are included in the $R_{V}$ given in Table I. The theoretical uncertainties in $R_{V}^{n}$ and $R_{V}^{(0)}$ are less than $1 \%$ and have a negligible impact on our analysis, so we do not quote these errors in Table I. The theoretical error in $\left(1-4 \sin ^{2} \theta_{W}\right)\left(1+R_{V}^{p}\right)$ is \pm 0.0008 [10], or slightly more than $1 \%$. Because this error receives roughly equal contributions from the uncertainty in $\hat{s}_{Z}^{2}$ and from the $\mathcal{O}(\alpha) Z \gamma$ box graph corrections, it is not appropriate to quote an uncertainty in $R_{V}^{p}$ alone. The uncertainties associated with other strong interaction effects are subdominant. For the kinematic range of the PV experiments analyzed here, the $R_{V}$ have a negligible impact on the $Q^{2}$ dependence of $A_{P V}^{p}$ and are taken to be constant. We use the conventional $\overline{M S}$ renormalization scheme. Therefore, $\sin ^{2} \theta_{W}$ in Eq. (2) and hereafter shall take its value as $\hat{s}_{Z}^{2}$.

For the reader's convenience, constant parameters (in some cases also the uncertainties) appearing in Eq. (2) and later in Eqs. (4) and (5) are summarized in Table I.

The effective axial form factor $G_{A}^{e}$ receives a number of contributions and may be written as

$$
\begin{aligned}
G_{A}^{e}\left(Q^{2}\right)= & G_{D}\left(Q^{2}\right)\left[\frac{g_{A}}{g_{V}}\left(1+R_{A}^{T=1}\right)\right. \\
& \left.+\frac{3 F-D}{2} R_{A}^{T=0}+\Delta s\left(1+R_{A}^{(0)}\right)\right],
\end{aligned}
$$

where

$$
G_{D}\left(Q^{2}\right)=\frac{1}{\left(1+Q^{2} / \Lambda_{A}^{2}\right)^{2}}
$$

parametrizes the $Q^{2}$-dependence with a dipole form and $\Lambda_{A}$ is the corresponding axial dipole mass. The ratio $-\frac{g_{A}}{g_{V}}$ is the isovector axial form factor of the nucleon at zero momentum transfer, which is precisely measured in the neutron $\beta$ decay. $F$ and $D$ are the octet baryon $\beta$ decay parameters, which can be determined by combining data from neutron and hyperon $\beta$ decays under the assumption of $\mathrm{SU}(3)$ flavor symmetry. $\Delta s$ is the strange quark contribution to nucleon spin. Assuming a gentle evolution from the perturbative to the nonperturbative domain, this quantity can be obtained from inclusive, polarized, deep inelastic lepton-nucleon scattering.

The ratios $R_{A}^{T=1}, R_{A}^{T=0}$, and $R_{A}^{(0)}$ characterize the effects of electroweak radiative corrections to the isovector, isoscalar, and $\mathrm{SU}(3)$ singlet hadronic axial vector amplitudes. Note that while $R_{A}^{T=1}$ and $R_{A}^{(0)}$ give the ratios of the $\mathcal{O}(\alpha)$ and tree-level hadronic axial vector neutral current amplitudes in the isovector and $\mathrm{SU}(3)$ singlet channels, respectively, $R_{A}^{T=0}$ does not have this interpretation because the tree-level isoscalar hadronic axial vector amplitude vanishes in the SM.

Conventionally, these quantities are divided into two pieces: the "one-quark" and "many-quark" contributions. The onequark contributions correspond to renormalization of the effective vector electron-axial vector quark couplings, $C_{2 q}$, and their values can be obtained from the SM predictions for these couplings given in Ref. [8]. The many-quark contributions include the so-called "anapole" effects as well as coherent strong interaction contributions to the radiative corrections. In contrast to the situation with the vector corrections, $R_{V}$, the relative importance of many-quark effects in the $R_{A}$ can be quite pronounced. The small vector coupling of the electron to the $Z$ boson, $g_{V}^{e}=-1+4 \sin ^{2} \hat{\theta}_{W} \sim-0.075$, leads to a suppression of the tree-level hadronic axial vector amplitude. However, $g_{V}^{e}$ is absent from a variety of both one- and many-quark radiative corrections. Thus, one would expect the magnitudes of the $R_{A}$ to be of the order of several percent, rather than the generic $\alpha / \pi \sim 0.3 \%$ scale normally associated with electroweak radiative corrections. As a result, the impact of otherwise negligible strong interaction effects in the many-quark corrections, such as the anapole contributions, can be amplified [14].

An appropriate framework for treating the many-quark effects associated with physics at the hadronic scale is chiral perturbation theory. A comprehensive analysis of the anapole contributions to $R_{A}^{T=1}$ and $R_{A}^{T=0}$ has been carried out to chiral order $p^{3}$ in Ref. [15]. This analysis included both one-loop contributions associated with the octet of pseudoscalar mesons as well as the full set of low-energy constants (LECs) that arise at this chiral order. A generous theoretical range was assigned to the LECs, leading to a quoted theoretical uncertainty in the total $R_{A}$ that is larger than the (logarithmically enhanced) one-quark corrections. The theoretical SM uncertainty is likely to be smaller.

The corresponding results, updated for the present value of the weak mixing angle, are given in Table II. The resulting prediction for $G_{A}^{e}$ is consistent with both the results of the SAMPLE deuterium measurement $[16,17]$, which is particularly sensitive to the dominant isovector axial component, 
TABLE II. The "one-quark" [8] and "many-quark" [15] corrections to the axial charges, both in $\overline{M S}$, as well as the combined corrections.

\begin{tabular}{llcc}
\hline \hline & \multicolumn{1}{c}{$R_{A}^{T=1}$} & $R_{A}^{T=0}$ & $R_{A}^{(0)}$ \\
\hline One-quark & -0.172 & -0.253 & -0.551 \\
Many-quark & $-0.086(0.34)$ & $0.014(0.19)$ & $\mathrm{N} / \mathrm{A}$ \\
Total & $-0.258(0.34)$ & $-0.239(0.20)$ & $-0.55(0.55)$ \\
\hline \hline
\end{tabular}

and the resultes of other theoretical models for the anapole contributions $[18,19]$. No evaluation of the many-quark contribution of $R_{A}^{(0)}$ has been made in the literature. We assume it is zero and assign the size of one-quark value for $R_{A}^{(0)}$ as its uncertainty.

The PV asymmetry for the neutron can be obtained by exchanging the $p$ and $n$ indices on the nucleon form factors in Eq. (2) and flipping the sign of the first isovector term in the expression for $G_{A}^{e}$ in Eq. (3). To first order, the PV asymmetry from a deuterium target is a cross-section weighted average of the proton and neutron asymmetries, which leads to an enhancement of the contribution of $G_{A}^{e}$ and a suppression to the relative contribution due to $G_{E}^{s}$ and $G_{M}^{s}$. Obviously a nuclear correction needs to be applied in the analysis. In this article, for the SAMPLE deuterium measurement, we adopt the asymmetry expression given in Ref. [16].
The ${ }^{4} \mathrm{He}$ nucleus is spin zero, parity even, and isoscalar. The PV asymmetry takes a much simpler form [4],

$$
A_{\mathrm{PV}}^{H e}=\frac{G_{F} Q^{2}}{4 \pi \sqrt{2} \alpha}\left(4 \sin ^{2} \theta_{W}\left(1+R_{V}^{T=0}\right)+\frac{2\left(1+R_{V}^{(0)}\right) G_{E}^{s}}{G_{E}^{p}+G_{E}^{n}}\right),
$$

where the isoscalar $R_{V}$ factor is related to $R_{V}^{p}$ and $R_{V}^{n}$ as

$$
R_{V}^{T=0}=\frac{R_{V}^{n}-\left(1-4 \sin ^{2} \theta_{W}\right) R_{V}^{p}}{4 \sin ^{2} \theta_{W}} .
$$

\section{EXPERIMENTAL DATA}

In this section, the world data of PV elastic scattering within a $Q^{2}$ range from 0.07 to $0.5(\mathrm{GeV} / \mathrm{c})^{2}$ is summarized. These include SAMPLE-H [17,20], SAMPLE-D [16,17], HAPPExH-99 [21], HAPPEx-H-a [22], HAPPEx-He-a [23], HAPPExH-b and HAPPEx-He-b [24], PVA4-H-a [25], PVA4-H-b [26], and the first $14 Q^{2}$ bins in $G^{0}$ forward angle [27]. The kinematics, the targets, and the measured asymmetries in these experiments are summarized in Table III. In column $A_{\text {phys }}$, the first and second uncertainties for the $G^{0}$ data are the uncorrelated and correlated experimental uncertainties, respectively. The values of $\eta_{E}$ and $\eta_{M}$ are also listed in the table. In calculating them, we have adopted a recent

TABLE III. A summary of the world data on PV elastic electron scattering within the range of $0.07(\mathrm{GeV} / \mathrm{c})^{2}<$ $Q^{2}<0.5(\mathrm{GeV} / \mathrm{c})^{2}$, including the average kinematics, targets, and published asymmetries $A_{\text {phys }}$, as well as coefficients $\eta_{E}$ and $\eta_{M}$. $A_{\text {phys }}, \eta_{E}$, and $\eta_{M}$ are in units of parts-per-million (ppm). The central kinematics for the two PVA4 measurements are obtained from Ref. [28]. For $A_{\text {phys }}$, the first and second uncertainties for the $G^{0}$ data are the uncorrelated and correlated experimental uncertainties, respectively.

\begin{tabular}{lccccccc}
\hline \hline Experiment & Target & $\begin{array}{c}Q^{2} \\
(\mathrm{GeV} / \mathrm{c})^{2}\end{array}$ & $\begin{array}{c}\theta_{\text {lab }} \\
(\mathrm{deg})\end{array}$ & $\begin{array}{c}A_{\text {phys }} \\
(\mathrm{ppm})\end{array}$ & $\begin{array}{c}\eta_{E} \\
(\mathrm{ppm})\end{array}$ & $\begin{array}{c}\eta_{M} \\
(\mathrm{ppm})\end{array}$ & Ref. \\
\hline SAMPLE-H & $\mathrm{H}_{2}$ & 0.1 & 144.4 & $-5.61 \pm 1.11$ & 2.07 & 3.47 & {$[17,20]$} \\
SAMPLE-D & $\mathrm{D}_{2}$ & 0.091 & 140.8 & $-7.77 \pm 1.03$ & 1.79 & 0.77 & {$[16,17]$} \\
HAPPEx-H-99 & $\mathrm{H}_{2}$ & 0.477 & 12.3 & $-15.05 \pm 1.13$ & 56.89 & 22.62 & {$[21]$} \\
HAPPEx-H-a & $\mathrm{H}_{2}$ & 0.099 & 6.0 & $-1.14 \pm 0.25$ & 9.55 & 0.76 & {$[22]$} \\
HAPPEx-H-b & $\mathrm{H}_{2}$ & 0.109 & 6.0 & $-1.58 \pm 0.13$ & 10.59 & 0.93 & {$[24]$} \\
HAPPEx-He-a & ${ }^{4} \mathrm{He}$ & 0.091 & 5.7 & $6.72 \pm 0.87$ & 20.19 & 0 & {$[23]$} \\
HAPPEx-He-b & ${ }^{4} \mathrm{He}$ & 0.077 & 5.8 & $6.40 \pm 0.26$ & 16.56 & 0 & {$[24]$} \\
PVA4-H-b & $\mathrm{H}_{2}$ & 0.108 & 35.52 & $-1.36 \pm 0.32$ & 10.08 & 1.05 & {$[26]$} \\
PVA4-H-a & $\mathrm{H}_{2}$ & 0.23 & 35.45 & $-5.44 \pm 0.60$ & 22.56 & 5.07 & {$[25]$} \\
$G^{0}$ & $\mathrm{H}_{2}$ & 0.122 & 6.68 & $-1.51 \pm 0.49 \pm 0.18$ & 11.96 & 1.17 & {$[27]$} \\
$G^{0}$ & $\mathrm{H}_{2}$ & 0.128 & 6.84 & $-0.97 \pm 0.46 \pm 0.17$ & 12.60 & 1.30 & {$[27]$} \\
$G^{0}$ & $\mathrm{H}_{2}$ & 0.136 & 7.06 & $-1.30 \pm 0.45 \pm 0.17$ & 13.46 & 1.48 & {$[27]$} \\
$G^{0}$ & $\mathrm{H}_{2}$ & 0.144 & 7.27 & $-2.71 \pm 0.47 \pm 0.18$ & 14.32 & 1.66 & {$[27]$} \\
$G^{0}$ & $\mathrm{H}_{2}$ & 0.153 & 7.50 & $-2.22 \pm 0.51 \pm 0.21$ & 15.31 & 1.89 & {$[27]$} \\
$G^{0}$ & $\mathrm{H}_{2}$ & 0.164 & 7.77 & $-2.88 \pm 0.54 \pm 0.23$ & 16.53 & 2.19 & {$[27]$} \\
$G^{0}$ & $\mathrm{H}_{2}$ & 0.177 & 8.09 & $-3.95 \pm 0.50 \pm 0.20$ & 17.99 & 2.58 & {$[27]$} \\
$G^{0}$ & $\mathrm{H}_{2}$ & 0.192 & 8.43 & $-3.85 \pm 0.53 \pm 0.19$ & 19.69 & 3.07 & {$[27]$} \\
$G^{0}$ & $\mathrm{H}_{2}$ & 0.210 & 8.84 & $-4.68 \pm 0.54 \pm 0.21$ & 21.77 & 3.71 & {$[27]$} \\
$G^{0}$ & $\mathrm{H}_{2}$ & 0.232 & 9.26 & $-5.27 \pm 0.59 \pm 0.23$ & 24.37 & 4.60 & {$[27]$} \\
$G^{0}$ & $\mathrm{H}_{2}$ & 0.262 & 9.92 & $-5.26 \pm 0.53 \pm 0.17$ & 28.00 & 5.99 & {$[27]$} \\
$G^{0}$ & $\mathrm{H}_{2}$ & 0.299 & 10.63 & $-7.72 \pm 0.80 \pm 0.35$ & 32.60 & 7.99 & {$[27]$} \\
$G^{0}$ & $\mathrm{H}_{2}$ & 0.344 & 11.46 & $-8.40 \pm 1.09 \pm 0.52$ & 38.40 & 10.89 & {$[27]$} \\
$G^{0}$ & $\mathrm{H}_{2}$ & 0.410 & 12.59 & $-10.25 \pm 1.11 \pm 0.55$ & 47.28 & 16.10 & {$[27]$} \\
\hline \hline
\end{tabular}


parametrization of the nucleon electromagnetic form factors from Ref. [29]. For the SAMPLE deuterium measurement, the $\eta_{M}$ is taken from Ref. [16], whereas its $\eta_{E}$ is taken to be 1.79 according to the static approximation.

\section{GLOBAL ANALYSIS}

\section{A. $A_{\text {nvs }}$ and theoretical uncertainties}

We now present a combined analysis of the world data aiming to extract $G_{E}^{s}$ and $G_{M}^{s}$. A global fit, generally speaking, is obtained by simultaneously solving a set of equations,

$$
m_{i} \pm \sigma\left(m_{i}\right)=t_{i}\left(a_{1}, a_{2}, \ldots\right) \pm \sigma\left(t_{i}\right),
$$

where $m_{i}$ and $t_{i}\left(a_{1}, a_{2}, \ldots\right)$, respectively, are the measured and theoretical values for experiment $i$. In this expression, $\sigma\left(m_{i}\right)$ and $\sigma\left(t_{i}\right)$ are their uncertainties, and $a_{1}, a_{2}, \ldots$ are the free parameters one seeks to determine. In our case,

$$
m_{i}=A_{\mathrm{phys}}^{i}, t_{i}=A_{\mathrm{nvs}}+\eta_{E} G_{E}^{s}+\eta_{M} G_{M}^{s},
$$

with $G_{E}^{s}$ and $G_{M}^{s}$ being the free parameters. In the previous section, we discussed the value and uncertainty of $A_{\text {phys }}^{i}$, as well as $\eta_{E}$ and $\eta_{M}$ (Table III). For each measurement, the values of $A_{\mathrm{nvs}}$ can be also computed straightforwardly using the formalism in Sec. I. They are listed in Table IV. We again have used the parametrization of the nucleon electromagnetic form factors from Ref. [29]. As mentioned, the $A_{\text {nvs }}$ for the SAMPLE deuterium measurement is calculated based on the asymmetry expression in Ref. [16] with the theoretical value of $G_{A}^{e}$.

The treatment of the theoretical uncertainty $\sigma\left(t_{i}\right)$ is more subtle. $\sigma\left(t_{i}\right)$ receives dominant contributions from the following sources: the nucleon axial form factor $\left(G_{A}^{e}\right)$, the nucleon electromagnetic form factors $\left(G_{E, M}^{(p, n)}\right)$, and the nuclear corrections. Theoretical uncertainties from a given source are correlated among different experiments. The uncertainty in $G_{A}^{e}$ can be calculated based on the uncertainties in Tables I and II. For the nucleon electromagnetic form factors, based on the spread of the world data (see, e.g., Ref. [29]), we estimate their relative uncertainties as

$$
\begin{aligned}
& \frac{\sigma\left(G_{E}^{p}\right)}{G_{E}^{p}}=2.5 \%, \quad \frac{\sigma\left(G_{M}^{p}\right)}{G_{M}^{p}}=1.5 \%, \\
& \frac{\sigma\left(G_{E}^{n}\right)}{G_{E}^{n}}=15 \%, \quad \text { and } \quad \frac{\sigma\left(G_{M}^{n}\right)}{G_{M}^{n}}=1 \%,
\end{aligned}
$$

respectively. This is consistent with the uncertainty assignment in Ref. [22], except that the uncertainty of $G_{E}^{n}$ in Ref. [22] was assigned more conservatively to be $30 \%$. Also note that we have made a simplifying assumption that these form factor uncertainties are "scaling" in nature, independent of the $Q^{2}$. For an analysis with relatively small range of $Q^{2}$, this is reasonable. Nuclear corrections are only relevant for nonhydrogen targets. For the two ${ }^{4} \mathrm{He}$ measurements, according to Ref. [24], 3\% is assigned as the fractional theoretical uncertainty of $A_{\mathrm{nvs}}$. Nuclear corrections for the SAMPLE

TABLE IV. A summary of $A_{\text {nvs }}$ and the theoretical uncertainties of individual measurements. The uncertainties are grouped by six different sources: $G_{A}^{e}, G_{E}^{p}, G_{M}^{p}, G_{E}^{n}, G_{M}^{n}$, and the nuclear corrections (N.C.) to ${ }^{4}$ He data. See text for details.

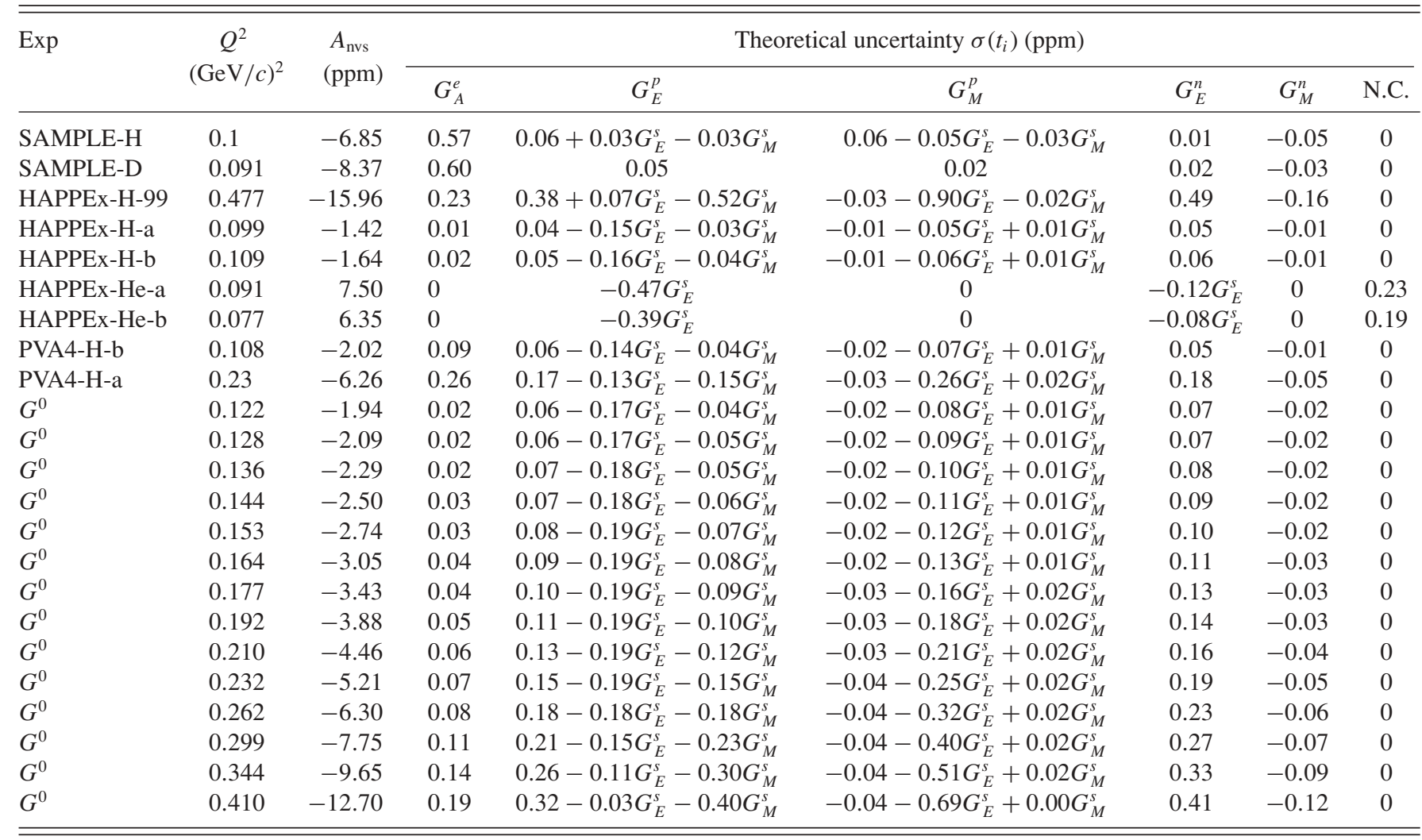


deuterium measurement have little impact on the final fit and are therefore neglected. The theoretical uncertainties for the $t_{i}$ in Eq. (8) due to the different sources are summarized in the last six columns in Table IV. To be precise, the content of each column gives the change in $A_{\mathrm{nvs}}+\eta_{E} G_{E}^{s}+\eta_{M} G_{M}^{s}$ when the source magnitude $\left(\left|G_{A}^{e}\right|,\left|G_{E, M}^{(p, n)}\right|\right.$, or nuclear correction to ${ }^{4} \mathrm{He}$ data) is increased by one standard deviation. Notice that the nucleon electromagnetic form factors also affect the value of $\eta_{E}$ and $\eta_{M}$ and therefore generate "pull terms" linear in $G_{E}^{s}$ and $G_{M}^{s}$ in Table IV. Such pull terms are neglected for the SAMPLE deuterium measurement.

\section{B. Combined analysis at $Q^{2}=0.1(\mathrm{GeV} / \mathrm{c})^{2}$}

As can be seen from Table III, a wealth of data exist with $Q^{2}$ in the vicinity of $0.1(\mathrm{GeV} / \mathrm{c})^{2}$, including SAMPLE-H, SAMPLE-D, HAPPEx-H-a, HAPPEx-H-b, HAPPEx-He-a, HAPPEx-He-b, PVA4-H-b, and low $Q^{2}$ data from $G^{0}$. It is natural to first make a combined analysis at $Q^{2}=0.1$. To interpolate all data to a common $Q^{2}$, we assume $G_{E}^{s} \propto Q^{2}$ and $G_{M}^{s}$ is a constant [30]. That is, we replace $\eta_{E} G_{E}^{s}\left(Q^{2}\right)+\eta_{M} G_{M}^{s}\left(Q^{2}\right)$ by $\tilde{\eta}_{E} G_{E}^{s}(0.1)+\eta_{M} G_{M}^{s}(0.1)$, where $\tilde{\eta_{E}}=\eta_{E} \frac{Q^{2}}{0.1(\mathrm{GeV} / \mathrm{c})^{2}}$. To simplify our notation, we use $G_{E}^{s}$ and $G_{M}^{s}$ hereafter to denote their values at $Q^{2}=0.1(\mathrm{GeV} / \mathrm{c})^{2}$. In the $\left(G_{E}^{s}, G_{M}^{s}\right)$ space, each measurement $i$ provides a linear constraint as

$$
\tilde{\eta}_{E}^{i} G_{E}^{s}+\eta_{M}^{i} G_{M}^{s}=A_{\mathrm{phys}}^{i}-A_{\mathrm{nvs}}^{i} .
$$

In Fig. 1, each constraint is shown as a linear band in the $\left(G_{E}^{s}, G_{M}^{s}\right)$ plane, where $\sigma\left(A_{\text {phys }}^{i}\right)$ (see Table III) and the theoretical uncertainty $\sigma\left(t_{i}\right)$ (see Table IV) have been combined in quadrature into an overall uncertainty. Somewhat arbitrarily, we include the three lowest $Q^{2}$ bins from the $G^{0}$ data in this part of the analysis. For visual clarity, they are combined into a single constraint as

$$
A_{\text {phys }}^{i}-A_{\mathrm{nvs}}^{i}=0.84 \pm 0.34=16.38 G_{E}^{s}+1.32 G_{M}^{s},
$$

which is shown as the solid black band in Fig. 1. From the figure, one sees that the agreement among different measurements is generally good. The $G^{0}$ and PVA4 appear to be offset from the HAPPEx-H-b measurement, but they nevertheless agree within $2 \sigma$. As explained in Sec. I, the SAMPLE deuterium measurement (dashed red band) has much less sensitivity to $G_{E}^{s}$ and $G_{M}^{s}$.

The ten different measurements (three from $G^{0}$, and the other seven from separate experiments) above provide redundancy in the joint determination of $\left(G_{E}^{s}, G_{M}^{s}\right)$. To solve for $\left(G_{E}^{s}, G_{M}^{s}\right)$ and determine the confidence contours, we follow the standard least square procedure (see, e.g., Ref [31]). Specifically, we rearrange Eq. (9) into the form of Eq. (7) as

$$
m_{i}=t_{i}\left(G_{E}^{s}, G_{M}^{s}\right) \pm \sigma_{i}+\sum_{k=0}^{6} \pm \beta_{i, k},
$$

where $m_{i}$ and $t_{i}$ are given in Eq. (8) and $\sigma_{i}$ is the uncorrelated experimental uncertainty. $\beta_{i, k}$ denotes the correlated uncertainty for measurement $i$ with "source index" $k$. In our case, $\beta_{i, 0}$ equals the correlated experimental uncertainty for the $G^{0}$ data and 0 for other experiments, and $\beta_{i, k=1,2,3,4,5,6}$ are

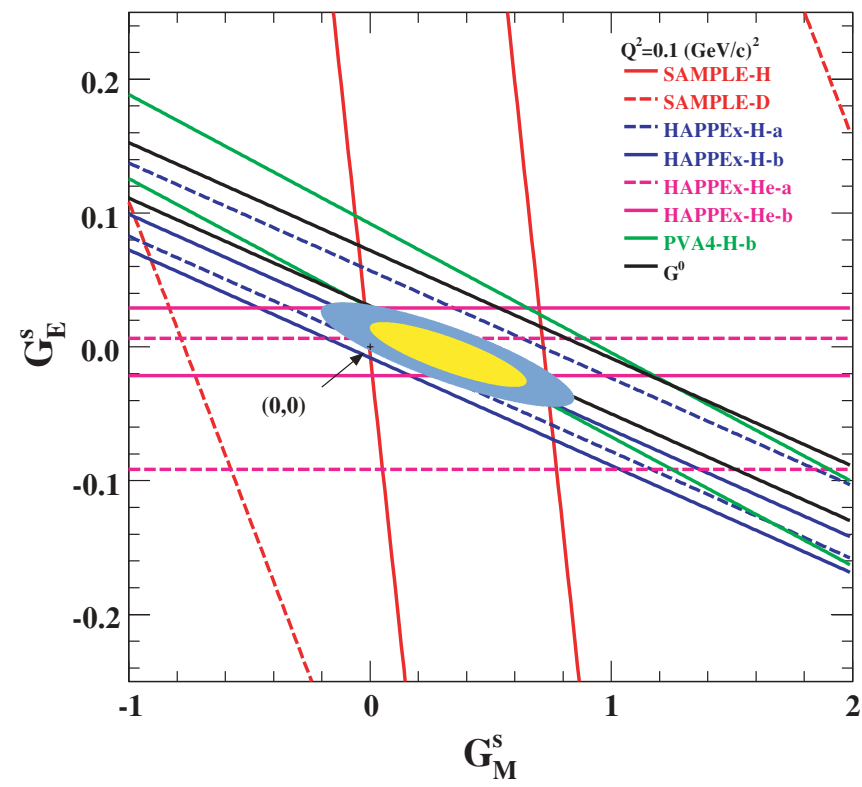

FIG. 1. (Color online) The world data constraints on $\left(G_{E}^{s}, G_{M}^{s}\right)$ at $Q^{2}=0.1(\mathrm{GeV} / \mathrm{c})^{2}$. The form factors of Kelly are used. Different bands in the plot represent SAMPLE-H [20] (solid red), SAMPLE-D [16] (dashed red), HAPPEx-H-a [22] (dashed blue), HAPPEx-H-b [24] (solid blue), HAPPEx-He-a [23] (dashed pink), HAPPEx-He-b [24] (solid pink), PVA4-H-b [26] (solid green), and the lowest three $Q^{2}$ bins in $G^{0}$ forward angle [27] (solid black). The yellow and gray blue (dark) ellipses represent $68.27 \%\left(\Delta \chi^{2}=2.3\right)$ and $95 \%$ $\left(\Delta \chi^{2}=5.99\right)$ confidence contours around the point of maximum likelihood at $\left(G_{E}^{s}=-0.006, G_{M}^{s}=0.33\right)$. The black cross represents $G_{E}^{s}=G_{M}^{s}=0$.

the correlated theoretical uncertainties for each measurement $i$ due to different sources (Table IV). Then for each given pair of $\left(G_{E}^{s}, G_{M}^{s}\right)$, the $\chi^{2}$ is calculated as

$$
\chi^{2}=\sum_{i} \sum_{j}\left(m_{i}-t_{i}\right)\left(V^{-1}\right)_{i j}\left(m_{j}-t_{j}\right),
$$

where $i$ and $j$ are indices of the measurements and $V$ is the variance matrix with $V_{i j}=\sigma_{i}^{2} \delta_{i j}+\sum_{k=0}^{6} \beta_{i, k} \beta_{j, k}$. It has been shown in Ref. [31] that the $\chi^{2}$ constructed this way satisfies the standard $\chi^{2}$ distribution, and the solution (best fit) can be found by minimizing this $\chi^{2}$. Applying this technique to the 10 measurements in Fig. 1, we obtain

$$
G_{E}^{s}=-0.006 \pm 0.016, \quad G_{M}^{s}=0.33 \pm 0.21,
$$

with a correlation coefficient of -0.83 between the two and a minimum $\chi_{\min }^{2}=9.90$ with 8 degrees of freedom. Note that the uncertainties above are $1 \sigma\left(\Delta \chi^{2}=1\right)$ "marginalized" uncertainties corresponding to the projections of the error ellipse onto the two axes. That is, for a given value of $G_{M}^{s}$, the $\chi^{2}$ is minimized by varying $G_{E}^{s}$ and vice versa. The range defined by this uncertainty for a given parameter corresponds precisely to $68.27 \%$ of the confidence interval of that parameter [32]. On the other hand, for the two parameters $\left(G_{E}^{s}\right.$ and $\left.G_{M}^{s}\right)$ that are jointly determined, the $68.27 \%$ confidence region is instead defined by $\Delta \chi^{2}=2.3$ contour [8]. To demonstrate the precision of the fit, we plot the $68.27 \%\left(\Delta \chi^{2}=2.30\right)$ and $95 \%\left(\Delta \chi^{2}=5.99\right)$ joint confidence levels in Fig. 1 
TABLE V. Results of the first order fit $\left(G_{E}^{s} \propto Q^{2}, G_{M}^{s}=\right.$ constant). Rows are ordered by the $Q^{2}$ of the data. Each row gives the results of the fit that includes all data in Table III up to, and including, the given $Q^{2}$. Row " $<0.11(\mathrm{GeV} / \mathrm{c})^{2}$ " represents the fit with low $Q^{2}$ data included up to PVA4-H-b. Columns " $\chi_{\min }^{2} / \nu$ " and "Prob" are the reduced $\chi^{2}$ and the probability $\left(\equiv \int_{\chi_{\min }^{2}}^{\infty} P_{\chi^{2}} d \chi^{2}\right)$ of the fit.

\begin{tabular}{lccccc}
\hline \hline$Q^{2}(\mathrm{GeV} / \mathrm{c})^{2}$ & Experiment & $G_{E}^{s}$ & $G_{M}^{s}$ & $\chi_{\min }^{2} / \nu$ & Prob $(\%)$ \\
\hline$<0.11(\mathrm{GeV} / \mathrm{c})^{2}$ & & $-0.008(0.016)$ & $0.26(0.22)$ & $4.7 / 5$ & 45.5 \\
0.122 & $G^{0}$ & $-0.007(0.016)$ & $0.27(0.22)$ & $4.9 / 6$ & 55.4 \\
0.128 & $G^{0}$ & $-0.006(0.016)$ & $0.30(0.22)$ & $8.4 / 7$ & 29.7 \\
0.136 & $G^{0}$ & $-0.006(0.016)$ & $0.33(0.21)$ & $9.9 / 8$ & 27.2 \\
0.144 & $G^{0}$ & $-0.007(0.016)$ & $0.30(0.21)$ & $13.0 / 9$ & 16.1 \\
0.153 & $G^{0}$ & $-0.007(0.016)$ & $0.30(0.21)$ & $13.0 / 10$ & 22.1 \\
0.164 & $G^{0}$ & $-0.008(0.016)$ & $0.29(0.21)$ & $13.8 / 11$ & 24.4 \\
0.177 & $G^{0}$ & $-0.010(0.016)$ & $0.24(0.21)$ & $19.5 / 12$ & 7.7 \\
0.192 & $G^{0}$ & $-0.010(0.016)$ & $0.22(0.21)$ & $20.3 / 13$ & 8.9 \\
0.210 & $G^{0}$ & $-0.012(0.016)$ & $0.19(0.21)$ & $22.1 / 14$ & 7.6 \\
0.230 & $\mathrm{PVA} 4$ & $-0.013(0.016)$ & $0.22(0.20)$ & $22.3 / 15$ & 10.1 \\
0.232 & $G^{0}$ & $-0.014(0.016)$ & $0.21(0.20)$ & $23.1 / 16$ & 11.0 \\
0.262 & $G^{0}$ & $-0.012(0.016)$ & $0.24(0.19)$ & $24.7 / 17$ & 10.2 \\
0.299 & $G^{0}$ & $-0.014(0.016)$ & $0.23(0.19)$ & $26.0 / 18$ & 10.0 \\
0.344 & $G^{0}$ & $-0.014(0.016)$ & $0.24(0.19)$ & $26.1 / 19$ & 12.7 \\
0.410 & $G^{0}$ & $-0.013(0.015)$ & $0.27(0.19)$ & $27.1 / 20$ & 13.1 \\
0.477 & $\mathrm{HAPPEx}-\mathrm{H}-99$ & $-0.015(0.015)$ & $0.25(0.19)$ & $28.6 / 21$ & 12.4 \\
\hline \hline
\end{tabular}

as the yellow (light) and gray (dark) ellipses, respectively. $\left(G_{E}^{s}, G_{M}^{s}\right)=(0,0)$ yields a $\Delta \chi^{2}$ of $4.81\left(\int_{4.8}^{\infty} P_{\chi^{2}} d \chi^{2}=9.0 \%\right.$, where $P_{\chi^{2}}$ is the $\chi^{2}$ probability distribution function [32]), which is depicted as the black cross in the figure.

\section{Inclusion of higher $Q^{\mathbf{2}}$ data from $G^{\mathbf{0}}$, PVA4, and HAPPEx}

The analysis presented so far has focused on the vicinity of $Q^{2}=0.1(\mathrm{GeV} / \mathrm{c})^{2}$. It is desirable to extend the analysis by including data at higher $Q^{2}$ from $G^{0}$, PVA4, and HAPPEx. As a first attempt, the previous assumption that $G_{E}^{s} \propto Q^{2}$ and $G_{M}^{s}$ is a constant was adopted. We refer to such a fit as the first order fit. Using the same $\chi^{2}$ construction as in Eqs. (11) and (12), we started by fitting the data in Table III with $Q^{2}<0.11(\mathrm{GeV} / \mathrm{c})^{2}$ (up to PVA4-H-b) and then systematically included more data in the fit with increasing $Q^{2}$. In Table $\mathrm{V}$, the resulting $\left(G_{E}^{s}, G_{M}^{s}\right)$ and the fit quality are summarized.

Compared to the results in Sec. III B, extending the fit to higher $Q^{2}$ gives consistent values for $G_{E}^{s}$ and $G_{M}^{s}$. One also notices that for $Q^{2}$ beyond $0.164(\mathrm{GeV} / \mathrm{c})^{2}$, the fit quality $\left(\chi_{\min }^{2} / \nu\right.$ and $\chi^{2}$ probability) deteriorates significantly, implying that our lowest order model is no longer able to capture the true $Q^{2}$ variation in these two quantities.

To better characterize the data at higher $Q^{2}$, therefore, one needs to introduce higher order $Q^{2}$ dependence to $G_{E}^{s}$ and/or $G_{M}^{s}$. For $\sqrt{Q^{2}}$ sufficiently below the mass of the kaon, chiral perturbation theory provides a systematic framework for characterizing the $Q^{2}$ dependence of nucleon form factors. In this context the strange magnetic moment, $\mu_{s} \equiv G_{M}^{s}(0)$, arises at chiral order $p^{2}$. The sub-leading $Q^{2}$ dependence of $G_{M}^{s}\left(Q^{2}\right)$ - sometimes called the strange magnetic radius-is nominally $\mathcal{O}\left(p^{4}\right)$ and is determined by a combination of chiral loop contributions and a corresponding LEC. Loop effects also generate an order $p^{3}$ contribution to the strange magnetic radius that is free of any new parameters $[33,34]$. However, this contribution is substantially canceled by the $\mathcal{O}\left(p^{4}\right)$ loop corrections, resulting in a strong dependence on the $\mathcal{O}\left(p^{4}\right)$ LEC [35]. In contrast, $G_{E}^{s}$ starts off at chiral order $p^{3}$, while the sub-leading $Q^{2}$ dependence (and corresponding LEC) arises at $\mathcal{O}\left(p^{5}\right)$.

Based on these considerations, we extend the previous analysis to include all known, sub-leading $Q^{2}$ dependence in $G_{M, E}^{s}$ through $\mathcal{O}\left(p^{4}\right)$. In practice, doing so amounts to including one new constant in our fit associated with the strange magnetic radius. Because we are interested in the implications for the values of $G_{M, E}^{s}$ at $Q^{2}=0.1(\mathrm{GeV} / \mathrm{c})^{2}$, we expand about this value of $Q^{2}$ rather than about $Q^{2}=0$, viz.,

$$
\begin{aligned}
G_{E}^{s}\left(Q^{2}\right) & =G_{E}^{s} \frac{Q^{2}}{0.1}, \\
G_{M}^{s}\left(Q^{2}\right) & =G_{M}^{s}+\mu_{s}^{\prime}\left(Q^{2}-0.1\right),
\end{aligned}
$$

where $G_{E}^{s}$ and $G_{M}^{s}$ again are used to represent their values at $Q^{2}=0.1(\mathrm{GeV} / \mathrm{c})^{2}$. This is referred to as the second order fit. As in the previous section, each row in Table III now leads to a new constraint in the form of Eq. (11), with $G_{E}^{s}$ and $G_{M}^{s}$ parametrized by Eq. (14). Again using Eqs. (11) and (12), we constructed a proper $\chi^{2}$ function and performed the least square fit for $\left(G_{E}^{s}, G_{M}^{s}, \mu_{s}^{\prime}\right)$ by including higher $Q^{2}$ data.

The results of this procedure are summarized in Table VI.

Several observations can be made from the table. First, compared to the first order fits, the additional free parameter $\mu_{s}^{\prime}$ does not improve the fit quality significantly. Reasonable fits can be obtained up to $Q^{2}$ of $0.210(\mathrm{GeV} / \mathrm{c})^{2}$, beyond 
TABLE VI. Results of the second order fit $\left(G_{E}^{s} \propto Q^{2}\right.$ and $G_{M}^{s}$ linear in $\left.Q^{2}\right)$. See the caption of Table V for a description of the table content. Row " $<0.11$ " represents the fit using low $Q^{2}$ data up to PVA4-H-b. The data at $Q^{2}=0.230$ and $0.477(\mathrm{GeV} / \mathrm{c})^{2}$ are from PVA4 and HAPPEx-H-99, respectively, and all others are from $G^{0}$. Uncertainties of $G_{E}^{s}, G_{M}^{s}$, and $\mu_{s}^{\prime}$ are "marginalized" $1 \sigma$ uncertainties. The last row represents the fit by using only the low $Q^{2}\left(<0.11(\mathrm{GeV} / \mathrm{c})^{2}\right)$ and HAPPEx-99 data.

\begin{tabular}{lcccrr}
\hline \hline$Q^{2}(\mathrm{GeV} / \mathrm{c})^{2}$ & $G_{E}^{s}$ & $G_{M}^{s}$ & $\mu_{s}^{\prime}(\mathrm{GeV} / \mathrm{c})^{-2}$ & $\chi_{\min }^{2} / \nu$ & Prob $(\%)$ \\
\hline$<0.11$ & $-0.002(0.017)$ & $0.37(0.25)$ & $-23.7(27.5)$ & $3.9 / 4$ & 41.3 \\
0.122 & $-0.006(0.017)$ & $0.30(0.24)$ & $-5.9(19.9)$ & $4.8 / 5$ & 43.6 \\
0.128 & $-0.011(0.017)$ & $0.20(0.23)$ & $15.7(13.9)$ & $7.1 / 6$ & 30.7 \\
0.136 & $-0.011(0.016)$ & $0.21(0.23)$ & $15.7(9.5)$ & $7.1 / 7$ & 41.4 \\
0.144 & $-0.007(0.016)$ & $0.30(0.22)$ & $0.1(6.9)$ & $13.0 / 8$ & 11.0 \\
0.153 & $-0.007(0.016)$ & $0.30(0.22)$ & $-0.1(5.3)$ & $13.0 / 9$ & 16.1 \\
0.164 & $-0.006(0.016)$ & $0.32(0.22)$ & $-2.3(4.0)$ & $13.5 / 10$ & 19.9 \\
0.177 & $-0.005(0.016)$ & $0.35(0.22)$ & $-5.8(2.7)$ & $15.0 / 11$ & 18.4 \\
0.192 & $-0.006(0.016)$ & $0.33(0.22)$ & $-4.2(2.0)$ & $15.7 / 12$ & 20.6 \\
0.210 & $-0.006(0.016)$ & $0.33(0.22)$ & $-3.6(1.4)$ & $15.9 / 13$ & 25.3 \\
0.230 & $-0.012(0.016)$ & $0.35(0.21)$ & $-1.5(1.0)$ & $20.1 / 14$ & 12.6 \\
0.232 & $-0.013(0.016)$ & $0.35(0.21)$ & $-1.6(0.9)$ & $20.2 / 15$ & 16.5 \\
0.262 & $-0.012(0.016)$ & $0.29(0.21)$ & $-0.4(0.7)$ & $24.4 / 16$ & 8.2 \\
0.299 & $-0.012(0.016)$ & $0.31(0.21)$ & $-0.6(0.6)$ & $24.8 / 17$ & 10.0 \\
0.344 & $-0.012(0.016)$ & $0.28(0.20)$ & $-0.3(0.5)$ & $25.7 / 18$ & 10.8 \\
0.410 & $-0.014(0.016)$ & $0.25(0.20)$ & $0.1(0.3)$ & $27.0 / 19$ & 10.4 \\
0.477 & $-0.014(0.015)$ & $0.28(0.20)$ & $-0.1(0.2)$ & $28.4 / 20$ & 10.1 \\
$<0.11$ and 0.477 & $-0.008(0.016)$ & $0.26(0.22)$ & $-0.3(0.3)$ & $4.7 / 5$ & 45.7 \\
\hline \hline
\end{tabular}

which the "flexibility" of our fit model again seems to be inadequate to describe the data. Second, the best values of $G_{E}^{s}$ and $G_{M}^{s}$ are very similar to those obtained from the first order fit (Table V). Third, the uncertainties of $G_{E}^{s}$ and $G_{M}^{s}$, as compared to those from the first order fit, are slightly larger due to the additional parameter $\mu_{s}^{\prime}$. Fourth, if we ignore the fit quality, and simply examine the mean and uncertainty of $\mu_{s}^{\prime}$, it is large and uncertain until the fit range goes up to $0.262(\mathrm{GeV} / \mathrm{c})^{2}$. This is expected, because it is difficult to determine the slope parameter with insufficient "lever arm." Also, fits beyond $0.262(\mathrm{GeV} / \mathrm{c})^{2}$ suggest a gentle $\mu_{s}^{\prime}$. [To illustrate this point, in the last row in Table VI we show the fit results by using only the low $Q^{2}\left(<0.11(\mathrm{GeV} / \mathrm{c})^{2}\right.$ and HAPPEx-99 data, which yield a consistent picture as described above.] However, the fit quality for $Q^{2}>0.210(\mathrm{GeV} / \mathrm{c})^{2}$ prevents us from making a strong statement about $\mu_{s}^{\prime}$ here.

For completeness, we also investigated the impact of including a $Q^{4}$ term in $G_{E}^{s}$ (corresponding to chiral order $p^{5}$ ). The resulting fit quality does not improve substantially with the inclusion of data with $Q^{2}$ beyond $\sim 0.2(\mathrm{GeV} / \mathrm{c})^{2}$, and both second order parameters in the form factors are poorly constrained. Nevertheless, the resulting $G_{E, M}^{s}$ are consistent with those obtained above.

Based on these considerations, we choose to use the first order fit up to $Q^{2}=0.164$ (Table V) as our final results in this analysis:

$$
G_{E}^{s}=-0.008 \pm 0.016, \quad G_{M}^{s}=0.29 \pm 0.21,
$$

with a correlation coefficient of -0.85 between the two and $\chi_{\min }^{2} / v=13.8 / 11$. Again, the uncertainties of the two form factors are marginalized $1 \sigma$ uncertainties corresponding to the projections of $\Delta \chi^{2}=1$ contour. This result is in good agreement with Eq. (13), for which only the lowest three $Q^{2}$ bins of $G^{0}$ data were included. For $G_{M}^{s}$ alone, the one-side confidence integral for a negative $G_{M}^{s}([-\infty, 0])$ is $12.3 \%$.

\section{CONCLUSION}

A combined analysis of the world PV electron scattering data has been performed to extract the nucleon strange electric and magnetic form factors $G_{E}^{s}$ and $G_{M}^{s}$ at $Q^{2}=$ $0.1(\mathrm{GeV} / \mathrm{c})^{2}$. Our treatment is similar to that of Ref. [36], but utilizes all available low $Q^{2}$ data including the recent HAPPEX results [22,23] and incorporates the uncertainties in the electromagnetic and axial form factors. We find that the agreement among different measurements is good and we obtain fits with acceptable $\chi^{2}$. Using a simple parametrization of the $Q^{2}$ variation in $G_{E}^{s}$ and $G_{M}^{s}$, a satisfactory global fit can be obtained up to a $Q^{2} \sim 0.2(\mathrm{GeV} / \mathrm{c})^{2}$. At $Q^{2}=$ $0.1(\mathrm{GeV} / \mathrm{c})^{2}$, the confidence integral for $G_{M}^{s}<0$ is $12.3 \%$, so substantially negative values of $G_{M}^{s}$ are highly disfavored by the fit. In addition, our best fit is consistent with $G_{E}^{s}=0$ at $Q^{2}=0.1(\mathrm{GeV} / \mathrm{c})^{2}$.

\section{ACKNOWLEDGMENTS}

The authors thank Professors Elizabeth J. Beise, Douglas H. Beck, and Jens Erler for very helpful discussions. This work was supported in part by National Science Foundation Grant PHY-0555674 and U. S. Department of Energy Contract DE-FG02-05ER41361. 
[1] D. Kaplan and A. Manohar, Nucl. Phys. B310, 527 (1988).

[2] R. D. McKeown, Phys. Lett. B219, 140 (1989).

[3] D. H. Beck, Phys. Rev. D 39, 3248 (1989).

[4] M. J. Musolf et al., Phys. Rep. 239, No. 1 (1994).

[5] For recent analyses of isospsin-breaking corrections, see Refs. [6,7].

[6] B. Kubis and R. Lewis, Phys. Rev. C 74, 015204 (2006).

[7] M. Viviani et al., arXiv:nucl-th/0703051.

[8] W. M. Yao et al., J. Phys. G 33, 1 (2006) and 2007 partial update for edition 2008.

[9] J. Erler, A. Kurylov, and M. J. Ramsey-Musolf, Phys. Rev. D 68, 016006 (2003).

[10] J. Erler and M. J. Ramsey-Musolf, Phys. Rev. D 72, 073003 (2005).

[11] H. Budd, A. Bodek, and J. Arrington, arXiv:hep-ex/0308005.

[12] B. W. Filippone and X.-D. Ji, Adv. Nucl. Phys. 26, 1 (2001).

[13] D. Adams et al. [Spin Muon Collaboration (SMC)], Phys. Rev. D 56, 5330 (1997).

[14] The one-quark contributions can be further enhanced by large logarithms associated with running between the weak and hadronic scales, leading to relative corrections of the order of $10 \%$ or more.

[15] S. L. Zhu, S. J. Puglia, B. R. Holstein, and M. J. Ramsey-Musolf, Phys. Rev. D 62, 033008 (2000).

[16] T. M. Ito et al., Phys. Rev. Lett. 92, 102003 (2004).

[17] E. J. Beise, M. L. Pitt, and D. T. Spayde, Prog. Part. Nucl. Phys. 54, 289 (2005).
[18] D. O. Riska, Nucl. Phys. A678, 79 (2000).

[19] C. M. Maekawa and U. van Kolck, Phys. Lett. B478, 73 (2000).

[20] D. T. Spayde et al. (SAMPLE), Phys. Rev. Lett. 84, 1106 (2000).

[21] K. A. Aniol et al. (HAPPEx), Phys. Rev. C 69, 065501 (2004).

[22] K. A. Aniol et al. (HAPPEx), Phys. Lett. B635, 275 (2006).

[23] K. A. Aniol et al. (HAPPEx), Phys. Rev. Lett. 96, 022003 (2006).

[24] A. Acha et al. (HAPPEx), Phys. Rev. Lett. 98, 032301 (2007).

[25] F. E. Maas et al. (PVA4), Phys. Rev. Lett. 93, 022002 (2004).

[26] F. E. Maas et al. (PVA4), Phys. Rev. Lett. 94, 152001 (2005).

[27] D. S. Armstrong et al. $\left(G^{0}\right)$, Phys. Rev. Lett. 95, 092001 (2005).

[28] J. Arvieux and F. E. Maas (private communications).

[29] J. J. Kelly, Phys. Rev. C 70, 068202 (2004).

[30] $G_{E}^{s}(0) \equiv 0$, because there is no net strangeness in nucleons.

[31] D. Stump et al., Phys. Rev. D 65, 014012 (2001).

[32] P. R. Bevington and D. K. Robinson, Data Reduction and Error Analysis for the Physical Sciences, second edition (McGrawHill, Boston, 1992).

[33] T. R. Hemmert, U. G. Meissner, and S. Steininger, Phys. Lett. B437, 184 (1998) [arXiv:hep-ph/9806226].

[34] T. R. Hemmert, B. Kubis, and Ulf-G. Meissner, Phys. Rev. C 60, 045501 (1999).

[35] H. W. Hammer, S. J. Puglia, M. J. Ramsey-Musolf, and S. L. Zhu, Phys. Lett. B562, 208 (2003).

[36] R. D. Young, J. Roche, R. D. Carlini, and A. W. Thomas, Phys. Rev. Lett. 97, 102002 (2006). 